\title{
Estudio de la degradación de los aceites de oliva sometidos a fritura. I. Determinación estadística del parámetro que mejor cuantifica esta degradación.
}

\author{
Por M. C. Ancin Azpilicueta y M. T. Martínez Remírez. \\ Laboratorio Municipal de Pamplona. San Saturnino 2. 31001 - PAMPLONA.
}

\section{RESUMEN}

Estudio de la degradación de los aceites de oliva sometidos a fritura. I. Determinación estadística del parámetro que mejor cuantifica esta degradación.

Al diseñar este experimento además de presuponer una homogeneidad inicial en los seis aceites estudiados, se controlaron todos los factores, de forma, que sólo hubiera una variable, la termooxidación, que afectase a la degradación.

La conclusión a la que se llegó, después del correspondiente tratamiento estadistico de los resultados, es que el parámetro fracción polar es el más específico y el que mejor mide la alteración de los aceites de oliva calentados.

PALABRAS-CLAVE: Aceite de oliva - Alteración termooxidativa - Estudio estadístico - Fracción polar.

\section{SUMMARY}

Study of degradation of olive oils subjected to thermoxidation. I. Statistical determination the parameter that best quantifies the degradation.

On designing this experiment, besides estimating the initial homogeneity of the six oils, which have been studied, all the factors are controlled in a way that only one variable namely, thermoxidation affects the degradation.

After the corresponding statistical treatment of the results had been carried out, the conclusion reached was that polar fraction parameter results to been the most specific, besides being the one that best measures the alteration of the heated olive oils.

KEY-WORDS: Olive oil - Polar fraction - Statistical study Thermooxidative alteration.

\section{INTRODUCCION}

En el calentamiento de las grasas se producen modificaciones en sus componentes que se incrementan notablemente si la operación se repite con la misma grasa.

Como ya es sabido, las alteraciones de las grasas y aceites responden a tres tipos de cambios:

a) Autooxidación: Definida por Perkins (1) como la oxidación de una grasa 0 aceite a temperaturas inferiores a $100^{\circ} \mathrm{C}$. b) Oxidación térmica: Es la que se produce en presencia de aire.

c) Polimerización térmica: Definida por el mismo autor como la oxidación que se produce entre 200 y $300^{\circ} \mathrm{C}$ en presencia del oxígeno.

Estas modificaciones originan compuestos a partir de los cuales se producen otros secundarios que pueden tener acción tóxica, entre estos últimos destaca la formación de compuestos polares (aldehídos, cetonas, ...) que originan cambios drásticos en la estructura primitiva del aceite.

En este sentido, una vez que ha empezado la hidrólisis, a partir del glicerol se origina la acroleína o propenal de elevado poder lacrimógeno y de acción intensa sobre la mucosa del aparato respiratorio, sin embargo la irritación que produce constituye señal de alarma para el usuario, por lo que es muy difícil que puedan contaminarse los alimentos fritos (2). A partir de los Acidos Grasos se producen una serie de compuestos denominados por Guillaumin "Especies químicas nuevas" (3), (4), (5), (6), (7), (8), y (9) y donde se incluyen los productos siguientes:

$$
\begin{aligned}
& \text { - ácidos conjugados. } \\
& \text { - monómeros cíclicos. } \\
& \text { - oxiácidos. } \\
& \text { - polímeros de oxidación. } \\
& \text { - polímeros térmicos. }
\end{aligned}
$$

En tanto que algunos autores como Dobarganes (10) y Varela y Cols. (11) han utilizado el análisis sensorial como complemento de índices habituales, para definir la calidad de la grasa y el momento en que debe ser desechada, otros como Gere (12), consideran que aún existiendo síntomas que indican deterioro (defecto en el olor y sabor, excesiva formación de humo y espuma, oscurecimiento del color, formación de residuos viscosos y resinosos, etc.), la evaluación sensorial es inadecuada como medida de calidad para los aceites fritos. 
Por todo ello, en el presente trabajo se ha realizado un estudio, en seis aceites de oliva comerciales, sometiéndolos a diferentes períodos de termooxidación, sin incluir ningún alimento y analizando los cambios en ellos producidos mediante índices físicos y químicos, análisis cromatográficos y determinación de compuestos polares y no polares (según el método oficial recomendado por la I.U.P.A.C., con la modificación de Dobarganes y cols. (1983) (13) y que proporciona mejores resultados que el método oficial.)

Todos los resultados se han obtenido por cuadruplicado para poder aplicarles los tratamientos estadísticos adecuados, que nos llevan a determinar con objetividad matemática el parámetro que mejor cuantifica la degradación del aceite sometido a termooxidación.

Los test estadísticos aplicados han sido dos, en primer lugar el Análisis de la Varianza o ANOVA. Con esta prueba se han comparado las dispersiones que presentan los datos: una es la dispersión debida al proceso de termooxidación que, por ser el "factor" que diferencia a las muestras, se denomina dispersión factorial, y la otra es la debida al azar, que se denomina dispersión de azar; posteriormente estas dispersiones se transforman en varianzas, y por último se comparan la varianza debida al factor (en nuestro caso la termooxidación), con la varianza debida al azar (variación debida al método en cada determinación). El cociente de ambas varianzas se compara con un valor teórico calculado por Snedecor, de esta comparación se concluye si es el factor o el azar el determinante de la modificación del parámetro.

El segundo test que se ha aplicado a los resultados es el de Newman-Keuls, con el que se realiza una comparación particularizada de las medias aritméticas de los resultados obtenidos para cada parámetro, para cada muestra y en cada momento de la termooxidación.

\section{PARTE EXPERIMENTAL}

\subsection{Muestras.}

Se ha partido de seis marcas de aceite de oliva comerciales, y sobre cada muestra se ha provocado, en las mismas condiciones experimentales, procesos de termooxidación sometiéndolas a calor durante 5 , $10,20,30,50,75$ y 100 horas, siendo los períodos de calentamiento de 5 horas.

Los recipientes empleados para la termooxidación han sido de vidrio pirex, no utilizándose recipientes metálicos para evitar el paso de cationes al aceite que actuarían como catalizadores del proceso degradativo, la relación superf./volumen es de $0,455 \mathrm{~cm}^{-1}$, el calentamiento se ha hecho en presencia de oxígeno, y en ausencia de cualquier alimento.
La temperatura a la que se sometió el aceite fue de $180^{\circ} \mathrm{C}$, utilizándose una placa calefactora con termostato para conseguir que dicha temperatura sea constante a lo largo del proceso.

\subsection{Determinaciones analiticas.}

\subsubsection{Test colorimétrico de orientación o} muestreo FRI-TEST.

Es un test sencillo y fácil de realizar, muy usado por los agentes oficiales de control en Suiza y la República Federal Alemana.

Está basado en la reacción, coloreada que desarrollan ciertos productos de oxidación en presencia de una solución alcalina.

El color que se obtiene en la reacción se compara con una gama de colores standard de una escala numerada convencionalmente del 1 al 4 .

La apreciación es la siguiente:

1.- Grasa de buena calidad poco o nada oxidada.

2.- Grasa oxidada se recomienda sustituir el baño de fritura en este momento.

3.- Grasa muy alterada, que no se puede usar.

4.- Grasa tan alterada que se corre el riesgo de producir fenómenos tóxicos en el consumidor.

\subsubsection{Absorción especifica en la zona ultravio-} leta.

Se ha llevado a cabo siguiendo la NORMA U.N.E. 55-057 modificada.

La técnica ha consistido en pesar una cantidad exacta de aceite en matraz aforado de $10 \mathrm{ml}$, disolver en ciclohexano de calidad para espectroscopía UVIR, y completar hasta enrase con el mismo disolvente.

Después se ha leido a las siguientes longitudes de onda 230, 232, 268 y $270 \mathrm{~nm}$.

Cálculos:

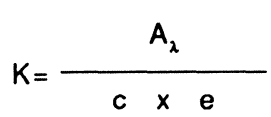

$$
\begin{aligned}
& \text { Siendo: } \\
& \mathrm{A}_{\lambda} \text { : Absorción leída. } \\
& \mathrm{c} \text { : Concentración de la } \\
& \text { disolución en } \mathrm{g} / 100 \\
& \mathrm{ml} . \\
& \mathrm{e}: \text { Espesor de la cubeta }
\end{aligned}
$$<smiles>[R10]C([Y10])P</smiles>
, los valores de $K_{232}$ y $K_{230}$ se relacionan con la formación de hidroperóxidos, dienos conjugados, compuestos carbonílicos y trienos conjugados, mientras que $K_{268} y$ $\mathrm{K}_{270}$ dependen de productos de oxidación secundarios, a partir de los compuestos iniciales, detectados en las anteriores longitudes de onda. 
2.2.3. Composición en ácidos grasos por cromatografía gaseosa.

Los ésteres metílicos preparados siguiendo la Norma U.N.E. 55-037-73 se cromatografían en un cromatógrafo VARIANT 3.700 , provisto de columna de $10 \%$ de DEGS sobre supelcoport $80 / 100$ de $2 \mathrm{~m}$ de longitud y $1 / 8^{n}$ de diámetro. Siendo la temperatura del inyector y detector $280^{\circ} \mathrm{C}$ y la temperatura de la columna $165^{\circ} \mathrm{C}$.

El gas portador es nitrógeno y su flujo de $30 \mathrm{ml} /$ min y está provisto de un registrador SHIMADZU C-R 1B CROMATOPAC.

\subsubsection{Determinación de compuestos polares.}

Se ha seguido el método recomendado por la I.U.P.A.C. excepto en la proporción éter de petróleo: éter etílico $(87: 13)(V: V)$, utilizada para el empaquetado de la columna y para la elución de la fracción no polar de los ésteres metílicos, usándose la mezcla (96:4) (V : V) modificación introducida por Dobarganes y cols. (13) en 1983 y que proporciona mejores resultados que la mezcla descrita por la I.U.P.A.C.

Esta técnica consiste en la separación de los compuestos polares y no polares por cromatografía en columna de silicagel 60 de la marca Merck de granulación y humedad bien definidos: partículas de tamaño $0,063-0,200 \mathrm{~mm}$ y una humedad del $5 \%$ $\mathrm{m} / \mathrm{m}$.

Para la determinación de compuestos polares y no polares del aceite en estudio, se empieza por preparar los ésteres metílicos según Norma U.N.E. 55-03773.

Posteriormente una cantidad de aproximadamente $0,5 \mathrm{~g}$ de dichos ésteres metílicos, se disuelven en la mezcla éter de petróleo: éter etílico (96:4) depositándola en la parte superior de la columna.

En primer lugar se eluyen los compuestos no polares con la mezcla éter de petróleo: éter etílico y después los polares con éter etílico.

Para comprobar que la separación ha sido total, se hace cromatografía en capa fina de ambas fracciones. Aplicándose en placas de silicagel de $0,2 \mathrm{~mm}$ de espesor y eluyéndose con una mezcla de éter de petróleo: éter etílico: ácido acético (80: 20:1) (V:V:V)

El revelado de las manchas se hace pulverizando con $\mathrm{SO}_{4} \mathrm{H}_{2}$ al $50 \%$ y posterior carbonización.

\subsubsection{Indices Físicos y Químicos.}

\subsubsection{Indice de acidez.}

Se ha hecho siguiendo la Norma U.N.E. 55-011

\subsubsection{Indice de lodo.}

De acuerdo con la Norma U.N.E. 55-013 usando la técnica de Hanus.

\subsubsection{Indice de Peróxidos.}

Según Norma U.N.E. 55-023-73.

\subsection{Métodos estadísticos.}

Para analizar con rigor los resultados se han aplicado dos test estadísticos diferentes, por una parte se ha usado el análisis de la varianza o ANOVA para un factor (en nuestro caso el proceso de termooxidación). Esta prueba debida a Fisher, es un proceso matemático que permite relacionar, indirectamente, varias medias aritméticas, obtenidas en diferentes muestras por medio de la comparación directa de sus dispersiones factorial y de azar.

Con este método estadístico se comprueba de forma global si las diferencias encontradas entre las medias pueden ser explicadas por el azar o si por el contrario son excesivas y deben atribuirse a la existencia de un factor, en nuestro caso el calentamiento, que hace que las medias se diferencien de forma significativa.

Sin embargo, aún habiendo comprobado la anterior relación, es conveniente cuantificar las diferencias encontradas entre las distintas medias, de los grupos horarios, determinando las parejas de datos que difieren significativamente de aquellas que no lo hacen. De los tratamientos matemáticos existentes a este tin hemos elegido para nuestro estudio el debido a Newman-Keuls.

Ambas pruebas estadísticas están ampliamente descritas en el manual de J.L. Carrasco (14).

\section{RESULTADOS Y DISCUSION}

A continuación en las tablas I, II, III, IV, V y $\mathrm{VI}$ se recogen los resultados de todos los parámetros medidos en los seis aceites estudiados, y en los diferentes períodos de termooxidación a que se ha sometido cada uno de ellos. 
Tabla 1

Variación de los valores de los parámetros estudiados, en la Muestra 1 de aceite de oliva, conforme avanza la termooxidación.

\begin{tabular}{|c|c|c|c|c|c|c|c|c|}
\hline & $\begin{array}{l}\text { Aceite } \\
\text { inicial }\end{array}$ & $\begin{array}{l}\text { 5h. } \\
\text { calen. }\end{array}$ & $\begin{array}{l}\text { 10r. } \\
\text { calen. }\end{array}$ & $\begin{array}{l}20 \mathrm{~h} . \\
\text { calen. }\end{array}$ & $\begin{array}{l}\text { 30h. } \\
\text { calen. }\end{array}$ & $\begin{array}{l}\text { 5oh. } \\
\text { calen. }\end{array}$ & $\begin{array}{c}75 b . \\
\text { calen. }\end{array}$ & $\begin{array}{l}\text { 100h. } \\
\text { calen. }\end{array}$ \\
\hline Fri - test & 1 & 1,5 & 2.5 & 3 & 3,5 & 4 & 4 & 4 \\
\hline $\begin{array}{l}\text { Acidez } \\
\text { g Oleico } \%\end{array}$ & $0,12 \pm 0,01$ & $0,21=0,00$ & $0,35 \pm 0,00$ & $0.51=0.01$ & $0,70 \pm 0,01$ & $0,71 \pm 0,01$ & $0,78 \pm 0,01$ & $1,16 \pm 0,01$ \\
\hline I. de Iodo & $81,59=0,72$ & $78,43 \pm 0,43$ & $78,55 \pm 0,29$ & $75,97 \pm 0,67$ & $72.99 \pm 0.23$ & $70,97 \pm 0,64$ & $61,13 \pm 1,33$ & $67,80 \pm 0,96$ \\
\hline $\begin{array}{l}\text { I.pefóxidos } \\
\text { meq } \\
2 / \mathrm{Kg} \text { grasa }\end{array}$ & $4,76 \pm 0,21$ & $5,21=0,44$ & $6,79 \pm 0,23$ & $7,62^{2} 0,27$ & $3,69 \pm 0,15$ & $4,64 \pm 0,27$ & $6,42 \pm 0,59$ & $5,26 \pm 0,14$ \\
\hline$K_{232}$ & 2,10 & 9,13 & 10,92 & 12,29 & 18,83 & 19,76 & 44,26 & 9.01 \\
\hline$K_{270}$ & 0,27 & 0,60 & 1,04 & 1,79 & 2,56 & 3,47 & 5,31 & 4,92 \\
\hline$* c_{18: 1}$ & $79,58 \simeq 0,20$ & $80,61 \pm 0,04$ & $79,81 \pm 0,21$ & $79,88^{I} 0,13$ & $79,56 \simeq 0,08$ & $79,17 \simeq 0,03$ & $79,56 \simeq 0,40$ & $78,60 \pm 0,17$ \\
\hline$* c_{18: 2}$ & $5.28 \pm 0.06$ & $4,27=0,07$ & $4,13 \pm 0,04$ & $3,06 \pm 0,09$ & $2,55 \simeq 0,05$ & $1,75 \pm 0,02$ & $1,27 \pm 0,03$ & $1,47 \pm 0,08$ \\
\hline \% F.Polar & $2,24 \pm 0,01$ & $10,27 \pm 1,97$ & $9,59 \simeq 1,07$ & $18,11 \pm 0,35$ & $17,52 \pm 0,98$ & $22,30 \pm 0,31$ & $24,55=1,50$ & $27,54 \pm 0,35$ \\
\hline \% F.no Polar & $92,00 \pm 3,97$ & $87,48 \pm 5,70$ & $89,22 \pm 1,10$ & $81,73 I_{2}, 12$ & $77,33 \div 0,78$ & $75,81 \pm 0,75$ & $73,57 \pm_{1,28}$ & $67,44 t_{4,10}$ \\
\hline
\end{tabular}

Tabla II

Variación de los valores de los parámetros estudiados, en la Muestra 2 de aceite de oliva, conforme avanza la termooxidación

\begin{tabular}{|c|c|c|c|c|c|c|c|c|}
\hline & $\begin{array}{l}\text { Aceite } \\
\text { Inicial }\end{array}$ & $\begin{array}{l}5 \mathrm{~h} . \\
\text { calen. }\end{array}$ & $\begin{array}{l}10 \mathrm{~h} . \\
\text { calen. }\end{array}$ & $\begin{array}{l}20 \mathrm{~h} . \\
\text { calen. }\end{array}$ & $\begin{array}{l}30 \mathrm{~h} \text {. } \\
\text { calen. }\end{array}$ & $\begin{array}{l}50 \mathrm{~h} . \\
\text { calen. }\end{array}$ & $\begin{array}{l}75 \mathrm{~h} . \\
\text { calen. }\end{array}$ & $\begin{array}{l}100 \mathrm{~h} . \\
\text { calen. }\end{array}$ \\
\hline Fri-test. & 1 & 1,5 & 2,5 & 3,5 & 4 & 4 & 4 & 4 \\
\hline $\begin{array}{l}\text { Acidez } \\
\text { g Oleicox }\end{array}$ & $0,39 \pm 0,01$ & $0,36 \pm 0,01$ & $0,38 \pm 0,00$ & $0,50 \pm 0,01$ & $0,73 \pm_{0}, 01$ & $0,59 \pm 0,01$ & $0,75 \pm 0,02$ & $1,17 \pm 0,03$ \\
\hline I. de Iodo & $79,89 \pm 0,34$ & $72,03 \pm 0,61$ & $78,38 \pm 0,40$ & $77,81 \pm 0,37$ & $74,20 \pm 0,75$ & $70,24 \pm 0,19$ & $62,68 \pm 0,32$ & $65,03 \pm 0,60$ \\
\hline 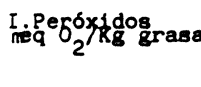 & a $9,15 \pm 0,18$ & $6,71 \pm 0,24$ & $10,33 \pm 0,27$ & $10,92 \pm 0,26$ & $4,93 \pm 0,20$ & $7,63 \pm 0,26$ & $3,29 \pm 0,42$ & $5,09 \pm 0,19$ \\
\hline$k_{232}$ & 2,22 & 5,86 & 7,58 & 14,05 & 19,82 & 21,22 & 36,94 & 14,36 \\
\hline$K_{270}$ & 0,46 & 0.49 & 0,74 & 2,22 & 3,32 & 3,59 & 5,02 & 2,02 \\
\hline$* C_{18: 1}$ & $80,24 \pm 0,05$ & $80,10 \pm 0,97$ & $80,51 \pm 0,09$ & $80,45 \pm 0,12$ & $80,88 \pm 0,09$ & $78,75 \div 0,14$ & $76,43 \pm 1,42$ & $74,68 \pm 9997$ \\
\hline * $C_{18: 2}$ & $4,27 \pm 0,04$ & $4,47 \pm 0,85$ & $3,88 \pm 0,18$ & $3,03 \pm 0,19$ & $2,26^{ \pm}-0,02$ & $1,14 \pm 0,03$ & $1,13 \pm 0,47$ & $1,11 \pm 0,20$ \\
\hline \% F.Polar & $6.50 \pm 0.03$ & $5,27 \pm 0,51$ & $7,70 \pm_{1,00}$ & $10,78 \pm 0,39$ & $13,77 \pm 3,01$ & $27,34 \pm 3,67$. & $41,26 \pm 1,07$ & $46,13 \pm 0,09$ \\
\hline * F.no Polar & $92,44 \pm 0,25$ & $90,57 \Psi_{1,72}$ & $90,60 \pm 3,05$ & $88,00 \pm 0,27$ & $84,23 \pm 2,71$ & $66,28 \pm_{3,51}$ & $56,51 I_{4,82}$ & $52,44 \pm 0,71$ \\
\hline
\end{tabular}


Tabla III

Variación de los valores de los parámetros estudiados, en la Muestra 3 de aceite de oliva, conforme avanza la termooxidación.

\begin{tabular}{|c|c|c|c|c|c|c|c|c|}
\hline & $\begin{array}{l}\text { Aceite } \\
\text { Inicial }\end{array}$ & $\begin{array}{l}5 \mathrm{~h} . \\
\text { calen. }\end{array}$ & $\begin{array}{l}10 \mathrm{~h} . \\
\text { calen. }\end{array}$ & $\begin{array}{l}20 \mathrm{~h} . \\
\text { calen. }\end{array}$ & $\begin{array}{l}30 \mathrm{~h} . \\
\text { calen. }\end{array}$ & $\begin{array}{l}50 \mathrm{~h} . \\
\text { calen. }\end{array}$ & $\begin{array}{l}75 \mathrm{~h} . \\
\text { calen. }\end{array}$ & $\begin{array}{l}100 \mathrm{~h} . \\
\text { calen. }\end{array}$ \\
\hline Fri-test & 1 & 1,5 & 2,5 & 3,5 & 4 & 4 & 4 & 4 \\
\hline Acjolez & $0,10 \pm 0,01$ & $0,15 \pm 0,00$ & $0,30 \pm 0,00$ & $0,55 \pm 0,02$ & $0,76 \pm 0,02$ & $0,88 \pm 0,00$ & $0,88 \pm 0,01$ & $1,21 \pm 0,05$ \\
\hline I. de Iodo & $82,98 \pm 0,67$ & $78,10 \pm 0,27$ & $77,35 \pm 0,68$ & $75,67 \pm 0,44$ & $72,62 \pm 0,09$ & $72,00 \pm 0,71$ & $67,55 \pm 0,60$ & $68,29 \pm 0,56$ \\
\hline $\begin{array}{l}\text { I. Peróxidos } \\
\text { meq } \mathrm{O}_{2} / \mathrm{Kg}\end{array}$ & grasa $3,28 \pm 0,09$ & $6,04 \pm 0,15$ & $9,16 \pm 0,15$ & $8,02^{ \pm 0} 0,31$ & $5,23 \pm 0,20$ & $8,73^{ \pm 0,29}$ & $3,79 \pm 0,18$ & $3,60 \pm 0,14$ \\
\hline$k_{232}$ & 2,36 & 9,65 & 13,18 & 10,16 & 15,46 & 19,40 & 23,29 & 18,25 \\
\hline$k_{270}$ & 0,20 & 0,64 & 1,08 & 1,27 & 4,80 & 5,22 & 9,88 & 3,37 \\
\hline * $c_{18: 1}$ & $78,59 \simeq 0,15$ & $80,44 \pm 0,96$ & $79,16 \pm 0,06$ & $80,06 \pm 0,06$ & $79,23 \pm 0,08$ & $79,58 I_{0,00}$ & $81,64 \pm 0,19$ & $78,19 \pm 0,70$ \\
\hline * $C_{18: 2}$ & $6,13 \pm 0,12$ & $4,65 \pm 0,14$ & $4,64 \pm 0,06$ & $3,49 \pm 0,65$ & $2,35 \pm 0,04$ & $1,82 \pm 0,24$ & $1,13 \pm 0,11$ & $1,97 \pm 0,06$ \\
\hline \% F.Polar & $3,30 \pm 0,60$ & $5,75 \pm 1,03$ & $7,97 \pm 0,58$ & $17,91 \pm 1,36$ & $18.20 t_{0}, 05$ & $27,17 \pm 2,96$ & $26,96 \pm 3,70$ & $28,30^{ \pm} 1,55$ \\
\hline * F.ne Polar & ir $96,56 \pm 0,27$ & $84,47 \pm 1,28$ & $90,75 \pm 2,04$ & $83,08 \pm 1,08$ & $79,05 \pm 1,27$ & $70,27 \pm 0,54$ & $71,20 \pm 0,74$ & $69,83^{I_{1}, 10}$ \\
\hline
\end{tabular}

Tabla IV

Variación de los valores de los parámetros estudiados, en la Muestra 4 de aceite de oliva, conforme avanza la termooxidación.

\begin{tabular}{|c|c|c|c|c|c|c|c|c|}
\hline & $\begin{array}{l}\text { Aceite } \\
\text { Inicial } \\
\end{array}$ & $\begin{array}{l}5 \mathrm{~h} . \\
\text { calen. }\end{array}$ & $\begin{array}{l}10 \mathrm{~h} . \\
\text { calen. }\end{array}$ & $\begin{array}{l}20 \mathrm{~h} . \\
\text { calen. }\end{array}$ & $\begin{array}{l}30 \mathrm{~h} . \\
\text { calen. }\end{array}$ & $\begin{array}{l}50 \mathrm{~h} . \\
\text { calen. }\end{array}$ & $\begin{array}{l}75 \mathrm{~h} . \\
\text { calen. }\end{array}$ & $\begin{array}{l}100 \mathrm{~h} . \\
\text { calen. }\end{array}$ \\
\hline Fri-test & 1 & 1,5 & 2,5 & 3 & 3 & 4 & 4 & 4 \\
\hline $\begin{array}{l}\text { Acidez } \\
\text { g Oleicox }\end{array}$ & $0,35 \pm 0,01$ & $0,35 \pm 0.01$ & $0,45 \pm 0,01$ & $0,60 \pm 0,01$ & $0,77 \pm 0,01$ & $0,91 \pm 0,03$ & $0,81 \pm 0,02$ & $1,21 \pm 0,10$ \\
\hline I. de Iodo & $81,08 \pm 1,03$ & $71,76 \pm 1,21$ & $79,25 \pm 0,53$ & $78,85 \pm 0,38$ & $75,55^{ \pm} 0,51$ & $72,55 \pm 0,43$ & 70,63 А, 49 & $66,87 \pm 0,71$ \\
\hline 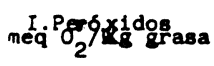 & a $7,92^{ \pm 0}, 31$ & $7,87 \pm 0,16$ & $9,03^{ \pm 0,28}$ & $9,24 \pm 0,89$ & $5,59 \pm 0,30$ & $5,12 \pm 0,45$ & $6,28 \pm 0,61$ & 4,55to, 31 \\
\hline$k_{232}$ & 2,10 & 5,90 & 11,97 & 13.26 & 17,21 & 18,52 & 24,81 & 17,61 \\
\hline$k_{270}$ & 0,18 & 0,70 & 0,89 & 1,81 & 3,90 & 4,39 & 6,92 & 3,87 \\
\hline$x c_{18: 1}$ & $78,41 \pm 0,33$ & $78,25 \pm 0,83$ & $79,06 \pm 0,01$ & $79,47 \pm 0,09$ & $79,16 \pm 0,12$ & $79,88 \pm 0,23$ & $77.84 \pm 0,48$ & $77,76 \pm 0,66$ \\
\hline$x c_{18: 2}$ & $6,11 \pm 0,15$ & $6,62 \pm 0,50$ & $5.00 \pm 0.02$ & $3,50 \pm 0,07$ & $3,17 \pm 0,02$ & $2,42 \pm 0,10$ & $2,02 \pm 0,21$ & 2,19,50, \\
\hline X F.Polar & $2,61 \pm 0,52$ & $4,73 \pm 0,44$ & $5.83 \pm 0.37$ & $14,80 \pm_{1,51}$ & $13,71 \pm 3,05$ & $21.05 \pm 1.31$ & $25,68 \pm 1,63$ & $28.96 \pm 1 . \infty$ \\
\hline X F.no Polar & $98,09 \pm 0,63$ & $96.77 \pm_{0,46}$ & $91,54 \pm_{1,19}$ & $82,44 \pm 1,85$ & $86,21 \pm 2,88$ & $74,81 \pm 1,50$ & $73,02^{ \pm 1,31}$ & $69,91 t_{1,10}$ \\
\hline
\end{tabular}


Tabla V

Variación de los valores de los parámetros estudiados, en la Muestra 5 de aceite de oliva, conforme avanza la termooxidación.

\begin{tabular}{|c|c|c|c|c|c|c|c|c|}
\hline & $\begin{array}{l}\text { Aceite } \\
\text { Inicial }\end{array}$ & $\begin{array}{c}5 \mathrm{~h} \\
\text { calen. }\end{array}$ & $\begin{array}{l}10 \mathrm{~h} . \\
\text { calen. }\end{array}$ & $\begin{array}{l}20 \mathrm{~h} \text {. } \\
\text { calen. }\end{array}$ & $\begin{array}{l}30 \mathrm{~h} \text {. } \\
\text { calen. }\end{array}$ & $\begin{array}{l}50 \mathrm{~h} . \\
\text { calen. }\end{array}$ & $\begin{array}{l}75 \mathrm{~h} . \\
\text { calen. }\end{array}$ & $\begin{array}{l}100 \mathrm{~h} . \\
\text { calen. }\end{array}$ \\
\hline Fri-test & 1 & 2,5 & 3 & 3,5 & 4 & 4 & 4 & 4 \\
\hline $\begin{array}{l}\text { Acjilez } \\
\text { goleox }\end{array}$ & $0.50 \pm 0,01$ & $0,41 \pm 0,01$ & $0,57 \pm 0,01$ & $0,60 \pm 0,01$ & $0,85 \pm 0,02$ & $0,78 \pm 0,00$ & $0,94 \pm 0,05$ & $1,27 \pm 0,02$ \\
\hline I. de Iodo & $82,38 \pm 1,14$ & $72,03 \pm 0,61$ & $83,23 \pm 0,76$ & $79,96 \pm 0,69$ & $78,06 \pm 0,74$ & $74,58 \tau_{0}, 37$ & $60,62^{I_{1}, 80}$ & $74,26 \pm 0,78$ \\
\hline $\begin{array}{l}\text { I.Peróxidos } \\
\text { meq } 0_{2} / K_{g} \text { grasa }\end{array}$ & $14,15 \pm 0,36$ & $4.78 \pm 0.21$ & $9,48 \pm 0,35$ & $5.55 \pm 0,28$ & $4,98 \pm 0,39$ & $6.87 \pm 0,62$ & $6,14 \div 0,26$ & $6,14 \pm 0,22$ \\
\hline $\mathrm{K}_{.232}$ & 1.92 & 8,42 & 9,88 & 12,19 & 19,52 & 20,92 & 20,93 & 6,30 \\
\hline $\mathrm{K}_{270}$ & 0,31 & 0,91 & 1.19 & 1,81 & 3,43 & 4,62 & 5,57 & 4,64 \\
\hline$* c_{18: 1}$ & $77,36 \pm 0,24$ & $77,98 \pm 0,92$ & $78,01 \pm 0,19$ & $78,61 \pm 0,23$ & $79,20 \pm 0,15$ & $78,94 \pm 0,08$ & $79,29 \pm 0,80$ & $77,09 \pm 0,72$ \\
\hline$\% c_{18: 2}$ & $7,53 \pm_{0}^{0,07}$ & $6.78 \pm 0.06$ & $6,50 \pm 0,03$ & $4,85 \pm 0,14$ & $4,04 \pm 0,36$ & $2,98 \pm 0,20$ & $2,19 \pm_{0,07}$ & $1,10 \pm 0,04$ \\
\hline \% F. Polar & $88,35 \pm 1,08$ & $91,50 \pm 0,33$ & $95,07 \pm 0,71$ & $8288 \pm 3,22$ & $82,34 \pm 4,65$ & $73,81 \pm 0,65$ & $70,49 \pm 0,34$ & $57,46 t_{1}, 13$ \\
\hline 6 F no Polar & $4,78 \pm 0,17$ & $6,99 \pm 0,64$ & $5,27 \pm 0,64$ & $14,32 \pm 0,20$ & $16,08 \pm 2,64$ & $20,20 \pm 0,47$ & $29,18 \pm 0,91$ & $37,42 \pm 1,60$ \\
\hline
\end{tabular}

Tabla VI

Variación de los valores de los parámetros estudiados, en la Muestra 6 de aceite de oliva, conforme avanza la termooxidación.

\begin{tabular}{|c|c|c|c|c|c|c|c|c|}
\hline & $\begin{array}{l}\text { Aceite } \\
\text { Inicial }\end{array}$ & $\begin{array}{c}5 \mathrm{~h} \\
\text { calen. }\end{array}$ & $\begin{array}{l}10 \mathrm{~h} . \\
\text { calen. }\end{array}$ & $\begin{array}{l}20 \mathrm{~h} . \\
\text { calen. }\end{array}$ & $\begin{array}{l}30 \mathrm{~h} . \\
\text { calen. }\end{array}$ & $\begin{array}{l}50 \mathrm{~h} . \\
\text { calen. }\end{array}$ & $\begin{array}{l}75 \mathrm{~h} . \\
\text { calen. }\end{array}$ & $\begin{array}{l}100 \mathrm{~h} . \\
\text { calen. }\end{array}$ \\
\hline Fri-test & 1 & 2 & 3 & 3,5 & 3,5 & 4 & 4 & 4 \\
\hline${ }_{\mathbf{8}}^{\text {Acjolez }}$ co \% & $0,70 \pm 0.00$ & $0,55 \pm 0,01$ & $0,42 \pm 0,00$ & $0,52 \pm 0,00$ & $0,70 \pm_{0,01}$ & $0,82 \pm 0,03$ & $0,87 \pm 0,03$ & $1,08 \pm 0,03$ \\
\hline I. de Iodo & $81,02 \div 0,24$ & $78,37 \pm 0,46$ & $78,51 \pm 0,33$ & $76,02 \pm 0,76$ & $72,86 \pm 0,18$ & $75,57 \pm 0,57$ & $67,00 \pm 0,66$ & $66,74 \pm 0,71$ \\
\hline $\begin{array}{l}\text { I. Peróxidos } \\
\text { meq } 0 / K_{\mathrm{K}} \text { grasa }\end{array}$ & $7,33 \pm 0,05$ & $5,80 \pm 0,27$ & $7,51 \pm 0,21$ & $9,33 \pm 0,80$ & $4,74 \pm 0,24$ & $7,36 \pm 0,18$ & $3,29 \pm 0,30$ & $3,42 \pm 0,15$ \\
\hline$k_{232}$ & 2,01 & 13,33 & 12,60 & 13,17 & 15,56 & 16,08 & 25,42 & 17,70 \\
\hline$k_{270}$ & 0,21 & 1,10 & 1,04 & 1,87 & 2,81 & 3,57 & 7,08 & 4,80 \\
\hline$x C_{18: 1}$ & $78,10 \pm 0,18$ & $81,61 \pm 1,14$ & $79,09 \pm 0,04$ & $79,09 \pm 0,09$ & $79,13 \pm 0,13$ & $78,99 \pm 0,19$ & $79,32 \pm 0,05$ & $77.69 \pm 0.82$ \\
\hline$* C_{18: 2}$ & $6,10 \pm 0,11$ & $3,67 \pm 0,66$ & $4,11 \pm 0,20$ & $3,19 \pm 0,13$ & $2.72 \pm 0.03$ & $2,13 \pm_{0}, 00$ & $1,19 \pm 0, \infty 0$ & $1,08 \pm 0,09$ \\
\hline X F.Polar & $3,03 \pm 0,77$ & $3,89 \pm 0,86$ & $5.21 \pm 0.58$ & $12,28 \pm 0,61$ & $15,10 \pm 1,23$ & $19,81 \pm 0,63$ & $30,23 \pm 0,97$ & $36,07 \pm 0.24$ \\
\hline X F.no Polar & $96,31 \pm 0,17$ & $93,14 \neq 1,79$ & $94,76 \pm 0,20$ & $81,31 \pm 0,36$ & $80,48 \pi, 96$ & $77,69 \pm_{1,63}$ & $62,84^{ \pm} 1,42$ & $62,64 \pm 0,76$ \\
\hline
\end{tabular}

Hay que resaltar que cada resultado es la media aritmética de las 4 mediciones que se han hecho, y cada media se expresa junto con el error.

De una visión global de estas tablas se observa que la acidez siempre aumenta conforme avanza la termooxidación, el índice de iodo disminuye debido a una ruptura de los dobles enlaces, el índice de peróxidos primero aumenta para disminuir posteriormente, ya que conforme avanza la termooxidación los peróxidos se transforman en otros compuestos como aldehídos, cetonas, etc.

En cuanto al porcentaje de linoleíco va disminu- 
Tabla VII

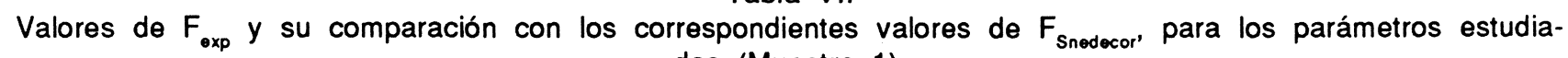
dos (Muestra 1).

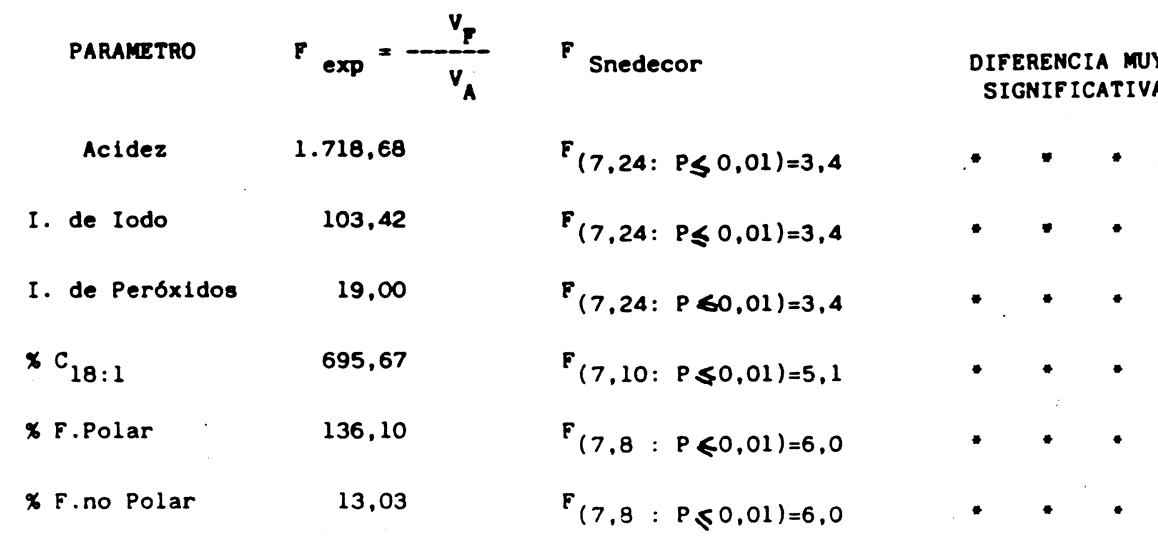

Tabla VIII

Valores de $F_{\text {exp }}$ y su comparación con los correspondientes valores de $F_{\text {Snedecor' }}$ para los parámetros estudiados (Muestra 2).

\begin{tabular}{|c|c|c|c|}
\hline PARAMETRO & $F_{\exp }=--\bar{v}_{A}^{-}$ & F Snedecor & $\begin{array}{l}\text { DIFERENCIA MUY } \\
\text { SIGNIF ICATIVA }\end{array}$ \\
\hline Acidez & 540,2 & $F(7,24: \quad P \leqslant 0,01)=3,4$ & - \\
\hline I. de Iodo & 190,77 & ${ }^{F}(7,24: P \leqslant 0,01)=3.4$ & $\cdot$ \\
\hline I.de Peróxidos & 151,00 & $F(7,24: P \leqslant 0,01)=3,4$ & - \\
\hline$x C_{18: 1}$ & 53,08 & $F(7,12: P \leqslant 0,01)=4,5$ & - \\
\hline \% F.Polar & 175,77 & $\left.F_{(7,8: P} \leq 0,01\right)=6,0$ & - \\
\hline * F.no Polar & 78,37 & $F(7,8: P \leqslant 0,01)=6,0$ & - \\
\hline
\end{tabular}

Tabla IX

Valores de $F_{\text {exp }}$ y su comparación con los correspondientes valores de $F_{\text {Snedecor }}$ para los parámetros estudiados (Muestra 3).

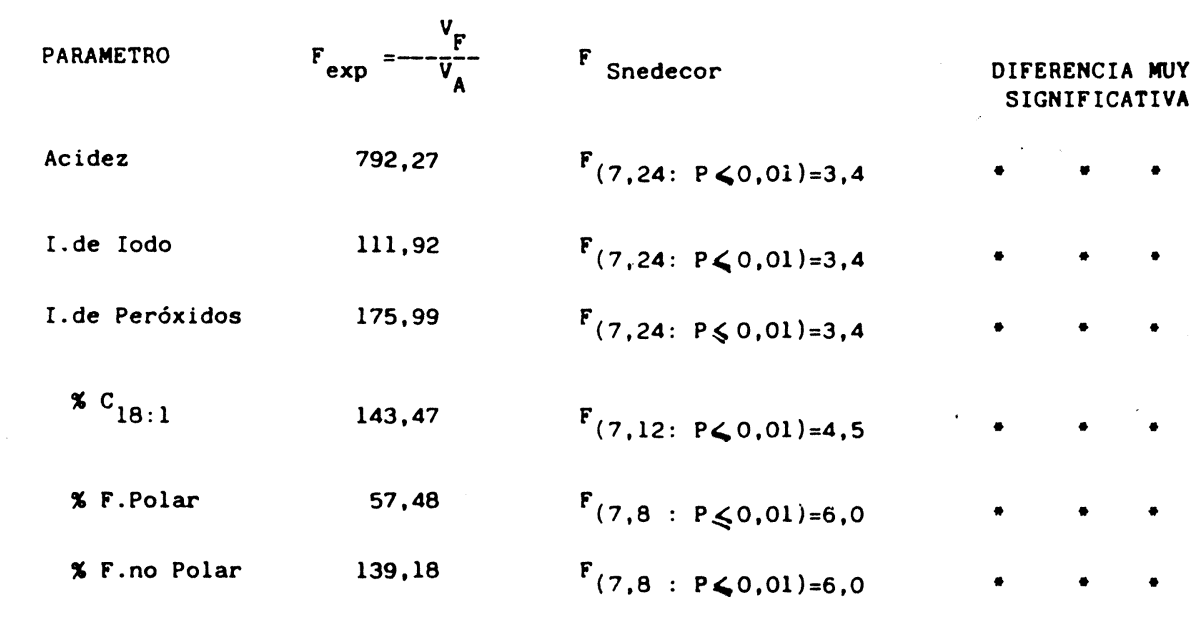


Tabla $X$

Valores de $F_{\text {exp }}$ y su comparación con los correspondientes valores de $F_{\text {snedecor }}$ para los parámetros estudiados (Muestra 4).

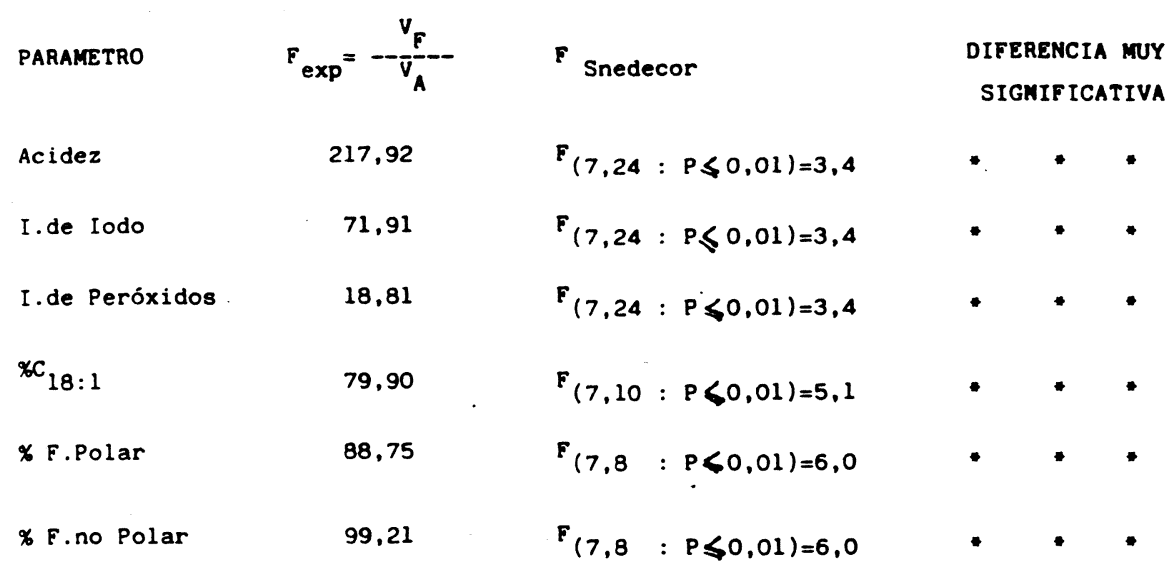

Tabla XI

Valores de $F_{\text {exp }}$ y su comparación con los correspondientes valores de $F_{\text {Snedecor }}$ para los parámetros estudiados (Muestra 5).

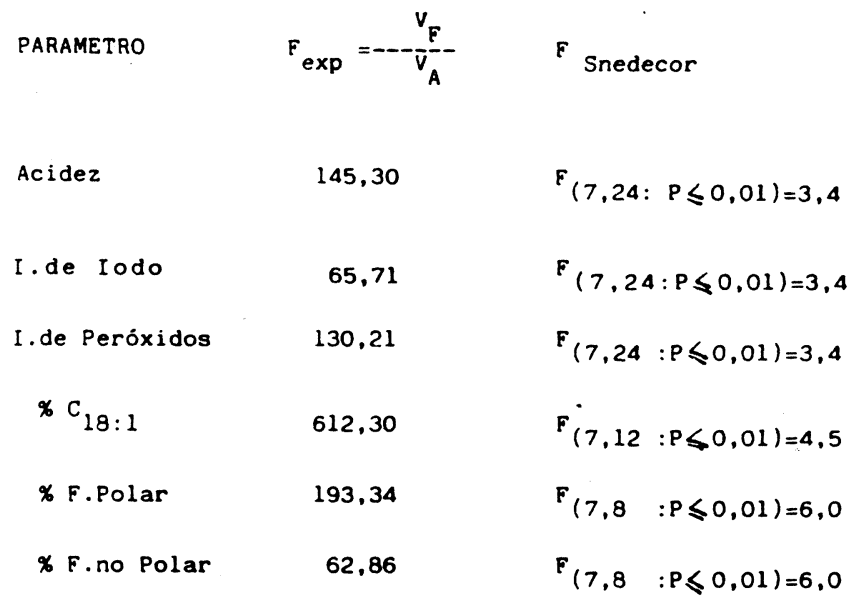

Tabla XII

Valores de $F_{\text {exp }}$ y su comparación con los correspondientes valores de $F_{\text {Snedecor }}$ para los parámetros estudiados (Muestra 6).

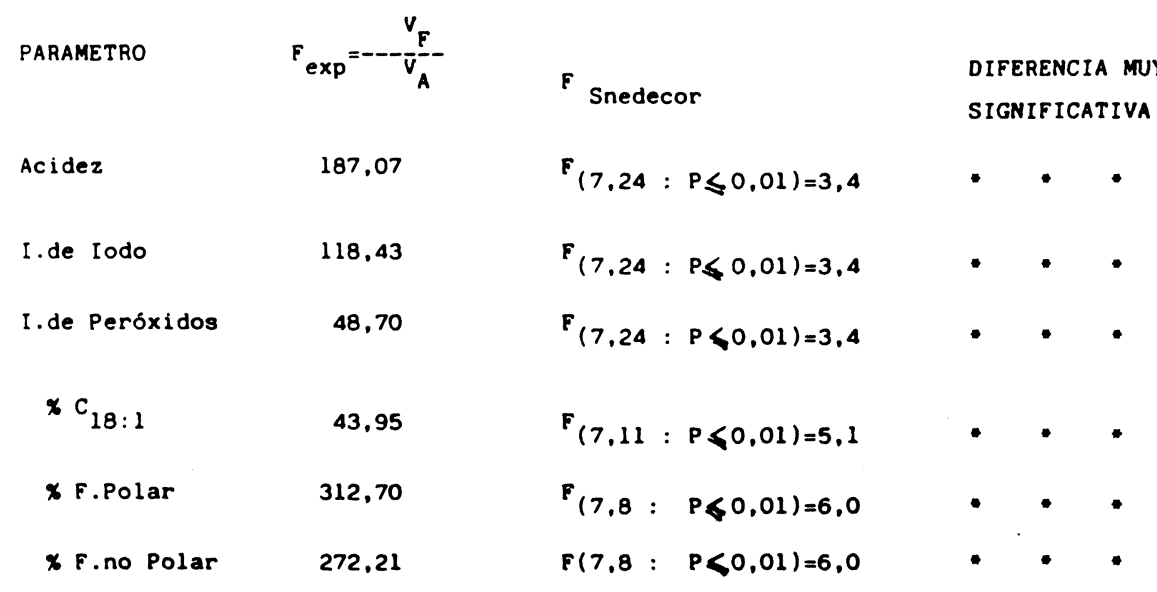


yendo, a diferencia del oleico que practicamente no sufre alteración, resultados que eran de esperar ya que el linoleíco tiene dos dobles enlaces, mientras que el oleico sólo tiene uno.

Por otra parte las fracciones polar y no polar varían de forma inversa, mientras la polar aumenta al avanzar la degradación del aceite la no polar va disminuyendo.

Para saber si la variación en el valor de estos parámetros se debe al azar, o si por el contrario, el factor termooxidación es el causante, se aplica el Análisis de la Varianza, que de forma rigurosa y con un grado de probabilidad o confianza, nos dice si las diferencias entre los datos son excesivas, en cuyo caso el azar no las puede explicar y por tanto se deben a un factor que en nuestro caso sería la termooxidación.

Recordemos que al diseñar este experimento presuponemos una homogeneidad inicial, en todo, excepto en el carácter que investigamos -termooxidación.

En las tablas VII, VIII, IX, X, XI y XII se recogen los valores de la $F$ exp y de la $F$ Snedecor de la acidez, índice de iodo, índice de peróxidos, porcentaje de linoleíco, porcentaje de fracción polar y porcentaje de fracción no polar, para cada una de las muestras estudiadas.

Se observa que en todos los casos, la $F$ exp $\neq F$ Snedecor para una $P \leq 0,01$. Por tanto existen diferencias significativas para todos los parámetros estudiados a lo largo del proceso de termooxidación. Concluyéndose que la termooxidación es el factor causal de la alteración de estos índices.

Posteriormente se aplicó el test de Newman-Keuls, y se comparó para cada pareja de datos la q experimental con la q teórica tabulada por Newman-Keuls, para observar entre qué fases del experimento aparecen diferencias significativas.

En el índice de acidez, índice de iodo e índice de peróxidos, se observa que las diferencias significativas para una $P \leq 0,01$ aparecen de forma irregular $y$ sin razón lógica aparente.

Sin embargo, cuando se aplica el test de Newman-Keuls en el porcentaje de linoleíco, se aprecia en los seis aceites de oliva estudiados que a partir de las cinco horas de calentamiento, este parámetro presenta diferencias significativas para una $P \leq 0,01$, con el aceite inicial, así como entre los distintos estadios de termooxidación.

En cuanto a la fracción no polar y polar de los ésteres metílicos de ácidos grasos, cuando se les aplica este test de Newman-Keuls, mientras la fracción no polar presenta un comportamiento irregular (debido a la retención variable que sufre en la columna de cromatografía) la fracción polar se comporta regularmente, apareciendo las diferencias significativas con el aceite inicial para una $\mathrm{P} \leq 0,01$ a partir de las 20 horas de termooxidación.

\section{CONCLUSIONES}

1.- El factor calor es en todos los casos estudiados, con una $P \leq 0,01$ el causante de la modificación que sufren los valores de los diferentes parámetros, a medida que avanza el proceso de termooxidación.

2.- No hay concordancia en el momento de sustituir el aceite, según los valores de Fri-test (criterio alemán) y los de acidez (criterio americano).

Así, mientras, según el Fri-test se debería sustituir el aceite a las 10 horas de termooxidación, ya que alcanza un valor superior a 2 , según los resultados del Indice de acidez, un aceite de oliva puede ser consumido hasta las 100 horas de calentamiento, momento en que alcanza el valor 1.

3.- Los parámetros de índice de iodo, índice de peróxidos, porcentaje de linoleíco y porcentaje de fracción no polar, además de admitir valores con intervalos de tolerancia muy amplios y de presentar gran variabilidad inicial en los aceites, tienen el inconveniente de ser difícilmente interpretables ya que al aplicarles el tratamiento estadístico, presentan diferencias significativas, para una $P \leq 0,01$, de forma irregular.

Esto confirma que su modificación no es específica de la alteración producida por la termooxidación.

4.- El comportamiento de la fracción polar de los ésteres metílicos de ácidos grasos, que en todos los casos presenta diferencias significativas para una $P \leq 0,01$ a partir de las 20 horas de calentamiento, sugiere que es la medida más objetiva para medir y cuantificar la termooxidación del aceite, y es la fracción que al aumentar hace que el aceite presente riesgo de toxicidad.

\section{AGRADECIMIENTO}

Agradecemos al Ilmo. Colegio Oficial de Farmacéuticos de Navarra la ayuda económica aportada para la realización de este trabajo. 


\section{BIBLIOGRAFIA}

1. Perkins, E. G.- "Nutritional and chemical changes occurring in heated fats: A review".- Food Technol. 10 (1960) 508514.

2. Champeix J.- "Les intoxications par l'acroleine".- Mason (Ed.) Lyon, 1967.

3. Chang y col.- "Chemical reactions involved in the deep-fat frying of foods".- J. Am. Oil Chemists'Soc. 55 (1978) 718-727.

4. Guillaumin, R. y col.- " Etude sur les huiles chauffées: Preparation et caracteristiques chimiques des huiles d'arachide palme, soja et tournesol chauffées a $200^{\circ} \mathrm{C}$ ".- Rev. Fr. Corps Gras 24 (1977) 477-481.

5. Viola, P.- "Les graisses dans l'alimentation humaine: L'huile d'o live".- Publicación Consejo Oleicola Internacional, 1978

6. Gray, J. J.- "Measurement of lipid oxidation a review".- J. Am. Oil Chemists'Soc. 55 (1978)539-546.

7. Permanyer, J. J. y col.- "Aceites calentados. Modificaciones fisicoquímicas de interés bromatológico. Estudio preliminar".- Anal. Bromatol. 29 (1977) 489-496.

8. Guillaumin, R- "Determination des espéces chimiques nouvelles formées durant le chauffage des huiles".- Rev. Fr. Corps Gras 20 (1973) 285-289.

9. Gere, A.- "Decrease in essential fatty acid and content of edible fats during the frying process".- Z. Ernahruhgswiss 21 (1982) 191-201.

10. Dobarganes Garcia, M. C.- "Aspectos generales de las grasas calentadas".- Grasas y Aceites 31 (1980) 417-422.

11. Varela, G., Moreiras-Varela, O. y Ruiz Roso, B.- "Utilización de algunos aceites en frituras repetidas. Cambios en las grasas y análisis sensorial de los alimentos fritos".- Grasas y Aceites 34 (1983) 101-107

12. Gere, A.- "A complex procedure for the analysis of used frying fats".- Fette Seifen Anstrich. 85 (1983) 111-117.

13. Dobarganes Garcia, M. C., Pérez Camino, M. C. y Gutierrez González-Quijano, R.-"Métodos analíticos de aplicación en grasas calentadas. I. Determinación de ésteres metílicos no alterados".- Grasas y Aceites 35 (1984) 172-177.

14. Carrasco, J. L.- "El método estadístico en la investigación médica".- Editorial Ciencia 3, 1983.

(Recibido: Mayo 1990) 